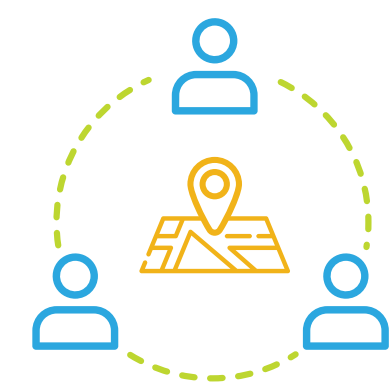

\title{
ARTICULAÇÃO REGIONAL: UMA ESTRATÉGIA PARA O ENFRENTAMENTO À SÍFILIS EM MUNICÍPIOS DA BAIXADA FLUMINENSE, ESTADO DO RIO DE JANEIRO
}

\author{
REGIONAL ARTICULATION: A STRATEGY FOR COPING WITH SYPHILIS IN \\ MUNICIPALITIES IN DOWNTOWN FLUMINENSE, STATE OF RIO DE JANEIRO
}

\begin{abstract}
Maria de Fátima Brito de Rezende
Graduada em Pedagogia pela Faculdade de Filosofia, Ciências e Letras de Volta Redonda - Fundação Rosemar Pimentel (FERP), Especialista em Gestão Hospitalar pela Escola Nacional de Saúde Pública Sergio Arouca - Fundação Oswaldo Cruz (ENSP-FIOCRUZ), Gestão de RH em Saúde (ENSP-FIOCRUZ), Gestão de Projetos de Investimentos em Saúde (ENSP-FIOCRUZ), Processos Educacionais - Metodologias Ativas pelo Hospital Sírio Libanês (HSL). Mestre em Educação Profissional em Saúde pela Escola de Saúde Pública Joaquim Venâncio - FIOCRUZ. Apoiadora do Projeto Resposta Rápida à Sífilis - Universidade Federal do Rio Grande do Norte (UFRN)/Ministério da Saúde (MS)/Organização Pan-Americana da Saúde (OPAS). Apoiadora do Conselho de Secretários Municipais de Saúde (COSEMS-RJ).

E-mail: fatrez.rezende3@gmail.com

Michelle Ribeiro de Sequeira

Cirurgiã dentista pela Universidade Federal do Rio de Janeiro (UFRJ), Especialista em Saúde da Família pela Escola Nacional de Saúde Pública Sergio Arouca - Fundação Oswaldo Cruz (ENSP-FIOCRUZ) e em Gestão da Atenção Básica (ENSP/FIOCRUZ). Mestre em Saúde Coletiva - Atenção Primária à Saúde (UFRJ). Apoiadora do Projeto Resposta Rápida à Sífilis Universidade Federal do Rio Grande do Norte (UFRN)/Ministério da Saúde (MS)

Organização Pan-Americana da Saúde (OPAS).

E-mail: michelle.sequeira@lais.huol.ufrn.br.
\end{abstract}

Paula Guidone Pereira Sobreira

Graduada em Psicologia pela Universidade Gama Filho (UGF), Especialista em Psicoterapia Infanto Juvenil Instituto Fernandes Figueiras - Fundação Oswaldo Cruz (IFF - FIOCRUZ). Mestre em Saúde Pública pela Escola Nacional de Saúde Pública Sergio Arouca - Fundação Oswaldo Cruz (ENSP-FIOCRUZ). Apoiadora do Projeto

Resposta Rápida à Sífilis Universidade Federal do Rio Grande do Norte (UFRN)/Ministério da Saúde (MS)/

Organização Pan-Americana da Saúde (OPAS). Professora e coordenadora de Pós-Graduação e

Extensão da Universidade Iguaçu (UNIG).

E.mail: paula.guidone@yahoo.com.br

Brena Gabriella Tostes de Cerqueira

Enfermeira pela Universidade Federal do Rio de Janeiro (UFRJ), Especialista em Saúde da Família pela Escola Nacional de Saúde Pública Sergio Arouca - Fundação Oswaldo Cruz (ENSP-FIOCRUZ) e em Gestão de Saúde pela Universidade do Estado do Rio de Janeiro (UERJ). Mestre em Gestão da Qualidade em Serviços de Saúde pela Universidade Federal do Rio Grande do Norte (UFRN). Gerente do Núcleo Estratégico e de Apoio Técnico da Fundação Estatal de Saúde de Niterói (FeSaúde).

E-mail: brena.fesaudeniteroi@gmail.com 


\section{Carlos Alberto Pereira de Oliveira}

Graduado em Didática Especial da Língua Inglesa pela Universidade do Estado do Rio de Janeiro (UERJ). Bacharel em Direito pela UERJ, advogado e Mestre em Educação pela Universidade Federal do Rio de Janeiro (1991). Professor visitante de Ética, Integridade e Condução Responsável da pesquisa da Universidade Federal de Mato Grosso do Sul (UFMS), membro do Comitê de Ética em Pesquisa da SMS/Rio. Bolsista do Laboratório de Inovação tecnológica em Saúde (LAIS) da Universidade Federal do Rio Grande do Norte e Vice-diretor do Instituto Multidisciplinar de Formação Humana com Tecnologias (IFHT) da UERJ. Membro do Comitê Executivo do International Council for Distance and Open Education (ICDE). Membro do Conselho Fiscal para o mandato de 2020/2022 da Associação Universidade em Rede (UNIREDE) eleito em 26 de setembro de 2019. Assessor

Externo Temporário para a Estratégia de Aprendizagem da Organização Mundial da Saúde (OMS).

E-mail: caoeduc@gmail.com

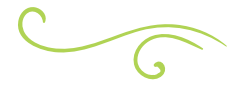

\section{RESUMO}

$O$ presente artigo traz para o debate a estratégia de articulação regional entre apoiadoras do Projeto "Sífilis Não" em oito municípios do Estado do Rio de Janeiro, contemplados pelas ações do Projeto Resposta Rápida à Sífilis nas Redes de Atenção. Esse projeto é de cunho federativo, fruto de cooperação técnica que envolve o Ministério da Saúde, Secretarias Estaduais e Municipais de Saúde, o Laboratório de Inovação Tecnológica em Saúde, como executor, a Secretaria de Educação a Distância e o Núcleo de Estudos em Saúde Coletiva da Universidade Federal do Rio Grande do Norte e a Organização Pan-americana de Saúde. A experiência contou com a participação/envolvimento de quatro apoiadoras, cada uma responsável pelo apoio em dois municípios distintos, localizados na Baixada Fluminense que apresentavam similaridades na organização dos serviços de saúde, vulnerabilidade política e social, entre outros aspectos. A ideia de organizar as ações a ser desenvolvidas regionalmente surgiu a partir do diagnóstico situacional realizado pelas apoiadoras nos seus respectivos municípios que, ao ser apresentado à gerência de Infecções Sexualmente Transmissíveis, com representação de diversas áreas técnicas da Secretaria Estadual de Saúde do Rio de Janeiro, evidenciou, a partir da apresentação dos nós críticos, a premência de uma articulação e instituição de um processo de trabalho conjunto para o enfrentamento das dificuldades enfrentadas.

Palavras-chave: Sífilis; Articulação regional; Estratégia. Processos de trabalho.

\section{ABSTRACT}

This report brings the strategy of regional articulation among supporters of the "Projeto Sífilis Não" in eight municipalities in the State of Rio de Janeiro, contemplated by the actions of the Projeto de Resposta Rápida à Sífilis nas Redes de Atenção. The result of technical cooperation involving the Ministry of Health, State and Municipal Health Secretariats, the Department of Distance Learning and the Center for Studies in Collective Health at the Federal University of Rio Grande do Norte (SEDIS and NESCUFRN) and the Organization Pan American Health Organization (PAHO) The experience included the participation / involvement of four supporters, each responsible for support in two different municipalities, located in the same health region that had similarities in the organization of health services, political, vulnerability and others. The idea of organizing the actions to be developed regionally arose from the situational diagnosis elaborated by the 
supporters in their respective municipalities, and then was presented to IST management, with representation of several technical areas of the State Health Secretariat - SES-RJ, the urgency of articulation and institution of a integrated work process became evident to face the imposed difficulties.

Key-words: Syphilis; Regional articulation; Strategy; Work process.

\section{INTRODUÇÃO}

OProjeto de Resposta Rápida ao Enfrentamento da Sífilis nas Redes de Atenção, nome fantasia Projeto "Sífilis Não" é um projeto de cunho interfederativo, fruto de cooperação técnica que envolve o Ministério da Saúde; Secretarias Estaduais e Municipais de Saúde; o Laboratório de Inovação Tecnológica em Saúde (LAIS) como executor, a Secretaria de Educação a Distância (SEDIS) e o Núcleo de Estudos em Saúde Coletiva (NESC) da Universidade Federal do Rio Grande do Norte; e a Organização Panamericana de Saúde (OPAS). Seu objetivo geral é "reduzir a Sífilis adquirida e em gestantes e eliminar a sífilis congênita no Brasil" (LUCAS et al., 2019, p. 11).

Sobre a sífilis no Brasil, nos últimos cinco anos, foi observado constante crescimento no número de casos de sífilis adquirida, em gestante e congênita, verificando-se que tanto a taxa de sífilis congênita, quanto a taxa de sífilis em gestantes triplicaram nesse período, passando de 2,4 para 6,8 e de 3,5 para 12,4 casos por mil nascidos vivos. Isso pode ter ocorrido pelo aumento da testagem e pelo aprimoramento do sistema de vigilância, entre outras determinantes (CERQUEIRA, 2018).

O Boletim Epidemiológico (BRASIL, 2020a) apresenta a comparação entre os anos de 2018/2019, observando reduções na taxa de detecção da sífilis em gestantes de 3,3\% e de 8,7\% na taxa de incidência da sífilis congênita. Em relação à sífilis adquirida, a redução foi de $4,6 \%$. As reduções verificadas ainda dependem de confirmações, uma vez que parte delas pode estar relacionada a problemas na transferência na base de dados entre as diferentes esferas de gestão do SUS (BRASIL, 2020a). Se confirmadas, podem ser atribuídas em parte ao Projeto Resposta Rápida à Sífilis nas Redes de Atenção, pela atuação da rede apoiadores nos municípios; e ao Ministério da Saúde, por ter pautado fortemente o enfrentamento da sífilis em todo o território nacional.

Quanto ao projeto, a investigação e o seguimento de casos de sífilis em gestantes nos municípios contemplados, essas são estratégias prioritárias desenvolvidas pela rede de apoiadores nesses municípios. Um eixo do Projeto a se considerar diz respeito às ações de Educação Permanente voltadas aos profissionais da rede de serviços de atenção à sífilis para a gestão e o cuidado integral das pessoas com sífilis, com destaque para a administração da penicilina, a ampliação da cobertura de testagem rápida nas Unidades de Atenção Primária de Saúde a partir de qualificação técnica das equipes para sua realização (BRASIL, 2020b).

A sífilis é uma Infecção Sexualmente Transmissível (IST) antiga, curável e aparentemente de fácil detecção e tratamento. No entanto, trata-se de um grave problema de saúde pública no Brasil e no mundo, reconhecido por vários autores. Sua erradicação depende, fundamentalmente, da vontade política e da disposição dos gestores locais em trazer para si e para sua pauta de gestão as ações de enfrentamento dessa problemática (LUCAS et al., 2019).

Cabe ainda à gestão local buscar a colaboração e a adesão dos profissionais de saúde e da sociedade civil, no sentido de organizar uma rede de apoio para o estabelecimento e a implantação de ações educativas e preventivas que deem conta de garantir melhor qualidade da atenção às gestantes e suas parcerias sexuais durante 0 pré-natal, de orientar e de informar os jovens e adultos em fase sexualmente ativa quanto aos riscos da infecção. Ademais, deve-se articular com as demais esferas de governo 
a disponibilização dos recursos/insumos necessários para a ampliação do acesso ao diagnóstico e ao tratamento (LUCAS et al., 2019). Dessa forma, entendemos que a erradicação da sífilis exige articulação, interação e envolvimento entre diferentes atores sociais e governamentais que, num movimento complexo e contínuo de ações de saúde, educação e vigilância, poderão reverter o quadro atual e garantir melhores condições de vida e saúde aos cidadãos.

Para a implantação do Projeto "Sífilis Não", foram selecionados 52 apoiadores institucionais, qualificados para atuação em 72 municípios brasileiros contemplados pelo referido projeto, formando, assim, uma rede de apoiadores institucionais que foram direcionados para atuação nos territórios a partir de março de 2018, quando iniciaram os primeiros contatos para identificação dos principais desafios a ser enfrentados e das potencialidades para o controle da sífilis nos municípios.

O Estado do Rio de Janeiro possui 92 municípios, divididos em nove regiões de saúde. Entretanto, no Projeto "Sífilis Não", foram contemplados quatro municípios da Região Metropolitana II, com duas apoiadoras; e nove municípios da Região Metropolitana I, com cinco apoiadoras. A chegada das apoiadoras no território fluminense foi semelhante ao da chegada dos demais apoiadores nos outros municípios contemplados pelo Projeto "Sífilis Não" no território brasileiro. Com muita disposição, sem nenhum contato prévio e nenhuma infraestrutura para realização do trabalho, cada um partiu para desbravar o seu território e construir o apoio necessário ao enfrentamento da sífilis a partir das orientações/diretrizes recebidas com maior ou menor grau de dificuldade, de acordo com sua trajetória profissional.

A Baixada Fluminense, território onde ocorreu a articulação regional para o enfrentamento da sífilis apresentada neste estudo, localiza-se na região Metropolitana I do Estado do Rio de Janeiro, primeira região de saúde do estado, instituída pela Lei
Complementar $\mathrm{N}^{\circ} 20$, de $1^{\circ}$ de julho de 1974 e se localiza entre as regiões do Médio Paraíba, Centro-Sul Fluminense e Serrana do estado do Rio de Janeiro (GOVERNO DO ESTADO DO RIO DE JANEIRO, 2018).

A Baixada Fluminense é composta por onze municípios, são eles: Belford Roxo, Duque de Caxias, Itaguaí, Japeri, Magé, Mesquita, Nilópolis, Nova Iguaçu, Queimados, São João de Meritie Seropédica. Desses, oito foram contemplados pelo Projeto Resposta Rápida à Sífilis, exceto, Itaguaí, Japeri e Seropédica, por terem população menor que 100.000 habitantes. Os oito municípios contemplados pelo projeto são próximos à capital do Estado, com alta densidade demográfica na sua grande maioria, expostos às desigualdades e vulnerabilidades sociais de todos os tipos, desde a distribuição desigual dos serviços e equipamentos urbanos, principalmente, na saúde até a crescente demanda por habitações com expansão de favelas trazendo como consequência a degradação ambiental, o aumento do desemprego, da exclusão social e da violência, características marcantes nos municípios da Baixada Fluminense.

O município que apresenta maior densidade demográfica é o de São João de Meriti, seguido pelos municípios de Nilópolis e Belford Roxo (GOVERNO DO ESTADO DO RIO DE JANEIRO, 2018). Em relação à cobertura da ESF ou da Atenção Básica, os municípios da Baixada Fluminense apresentam baixa cobertura, o que se reflete em grandes dificuldades de acesso da população aos serviços de saúde.

Numa análise mais geral, a Baixada Fluminense possui um dos piores indicadores de pobreza e renda. Sua população convive com enchentes urbanas, deslizamentos de encostas, empobrecimento dos solos, poluição dos rios, falta de água, baixa capacidade instalada de equipamentos de saúde, resultado do processo desordenado da ocupação humana e industrial no território, agravado pelo descaso dos governantes. 
A chegada das apoiadoras aos territórios iniciou com a apresentação do Projeto aos gestores municipais e áreas técnicas dos municípios, ao COSEMS-RJ, ao gestor e à área técnica da Secretaria Estadual de Saúde (SES-RJ) e aos Conselhos Municipais de Saúde. Também foram organizadas as agendas de reuniões presenciais nos territórios. Assim, demos início ao diagnóstico situacional analisando os fluxos e processos de trabalho das equipes, o que nos permitiu classificar os nós críticos em três eixos: estrutura, recursos humanos e gestão da clínica.

A necessidade de buscar uma articulação regional justificava-se pela conformação regional de saúde da Metropolitana I e sua divisão político-administrativa, bem como os nós críticos identificados por cada apoiadora em seus territórios de atuação, que evidenciaram a urgência de articulação e instituição de um processo de trabalho conjunto para o enfrentamento das dificuldades impostas por esses municípios, uma vez que a partir do diagnóstico situacional foram aferidas similaridades entre fragilidades, vulnerabilidades, desafios e potencialidades em alguns municípios que exigiam ações conjuntas para os seus enfrentamentos.

Nesse sentido, o presente relato tem por objetivo evidenciar a experiência de articulação regional como estratégia para o enfrentamento da sífilis nos municípios da Baixada Fluminense - RJ, desenvolvida pelas apoiadoras do Projeto "Sífilis Não", entre a equipe gestora de diferentes áreas técnicas da saúde dos municípios que compõem a referida região de saúde e com a equipe técnica da SES-RJ, envolvendo as áreas técnicas de Atenção Básica, Vigilância Epidemiológica em Saúde, Gerência de IST/HIV, AIDS e Hepatites Virais, Saúde da Mulher, Saúde da Criança, que culminou com a realização de quatro oficinas regionais no período de dezembro de 2018 a dezembro de 2019.

\section{MÉTODO}

No primeiro momento, as apoiadoras apresentaram o Projeto "Sífilis Não" aos gestores municipais e áreas técnicas dos municípios, assim como buscaram a sensibilização para a adesão ao Projeto e o estabelecimento de agendas de reuniões presenciais com representação das áreas técnicas (Planejamento, Atenção Primária, Vigilância, Saúde da Mulher, da Criança, Saúde do Homem e Saúde na Escola), de acordo com a organização dos serviços de saúde no município para discussão e construção do diagnóstico situacional.

A proposta de juntar, numa mesma reunião, as representações das diferentes áreas técnicas que atuam nos municípios teve como objetivo promover a articulação entre elas e buscar a consolidação do desenvolvimento do projeto "Sífilis Não", a partir do trabalho em equipe, cujo objetivo era contribuir com a construção de planos e ações que fossem apropriados pelas equipes e, assim, pudessem se estruturar de forma a compor os processos de trabalhos de cada área técnica, avaliados e revisados por eles e pelos profissionais que compõem suas equipes, independentemente da presença do apoiador. Pensamento este que se fundamenta nas caraterísticas do trabalho em equipe apresentadas pelos autores Robbins e Finley (1997). Para eles, a definição do trabalho em equipe apresenta algumas características, entre elas, o compromisso com as metas e os objetivos da equipe, o enfrentamento de conflitos existentes, o respeito às diferenças de pensamento. Esse entendimento proporcionou às apoiadoras do projeto uma melhor compreensão sobre a importância do engajamento das equipes nos processos de trabalho para o desenvolvimento das ações a ser implantadas pelo projeto. Dessa forma, todos podem contribuir com suas ideias, pensamentos, opiniões e ações para a solução dos problemas a ser enfrentados num contínuo processo de avaliação/alterações das ações e estratégias em desenvolvimento (ROBBINS; FINLEY, 1997). 
Com o objetivo de traçar um diagnóstico inicial, já nos primeiros contatos com as representações das áreas técnicas municipais, equipe designada pelos gestores para a discussão da implantação do Projeto "Sífilis Não", no período entre maio e junho de 2018, as apoiadoras disponibilizaram um questionário contendo dez questões a ser respondidas pelas áreas técnicas (vide figura 1). As respostas recebidas pelas apoiadoras possibilitaram um diagnóstico inicial como disparador das discussões e da elaboração do plano de ações a ser desenvolvido no território.

Nesse mesmo período, a supervisão do Projeto veio ao Rio de Janeiro para fazer uma apresentação para as áreas técnicas da SES-RJ com participação de todas as apoiadoras, organizada pelo contato da gerência de IST/ AIDS do MS com a gerência de IST/AIDS da SES-RJ. Na ocasião, as apoiadoras comentaram sobre o diagnóstico inicial, incluindo a análise dos instrumentos de gestão que estava em andamento nos municípios. A partir de então, o encaminhamento dado para a reunião seguinte foi a apresentação da análise dos instrumentos gestão/planejamento (RAG - Relatório Anual de Gestão; PAS Programação Anual de Saúde; PMS - Plano Municipal de Saúde e Plano de Enfrentamento da Sífilis), assim como as ações em desenvolvimento e principais dificuldades encontradas pelas apoiadoras. Para essa apresentação, as apoiadoras trabalharam com um formato padrão que foi apresentado à equipe técnica da SES-RJ um por região, separando a Região Metropolitana I da Região Metropolitana II.

Na apresentação da Metropolitana I, ficou evidenciado que a conformação, organização e estruturação dos serviços de saúde do município do Rio de Janeiro, sede da capital, em muito se diferenciava dos municípios da Baixada Fluminense, cuja conformação, estrutura e organização dos serviços apresentavam fortes similaridades quanto a desafios, fragilidades/potencialidades e vulnerabilidades. Essa diferenciação estrutural entre a capital e esses municípios trouxe uma implicação para as apoiadoras dada à similaridade do contexto de saúde encontrado na Baixada Fluminense, o que as levou a discutir uma estratégia de enfrentamento conjunto e identificar a problemática junto à coordenação do apoio que aprovou a ideia. Com isso, as apoiadoras dos municípios afins passaram a se reunir sistematicamente para discutir as ações que poderiam ser desenvolvidas conjuntamente, assim como os encaminhamentos necessários.

Como parte fundamental do estabelecimento de um processo de trabalho em conjunto, as apoiadoras fizeram, junto às equipes técnicas dos municípios, o levantamento, a avaliação e a discussão dos nós críticos identificados em cada município a partir do questionário aplicado e das dificuldades encontradas para compor um diagnóstico situacional de saúde, de forma a indicar um direcionamento para o apoio institucional no enfrentamento à sífilis na Baixada Fluminense.

A classificação dos nós críticos identificados deu-se a partir de três eixos distintos, quais sejam: estruturais, recursos humanos e gestão da clínica (vide figura 2 - eixos/nós críticos). O nó crítico do eixo estrutural contemplou capacidade instalada, a cobertura populacional dos serviços de saúde, a estrutura física, a oferta de serviços e os insumos necessários ao enfrentamento da sífilis, as fragilidades nas articulações/comunicações entre serviços/equipes. $\bigcirc$ nó crítico relacionado aos recursos humanos buscou tratar da formação/organização das equipes profissionais considerando o quantitativo existente em cada unidade de saúde, déficit, fragilidade/precarização contratual, alta rotatividade profissional, qualificação técnica, entre outros aspectos. O eixo gestão da clínica identificou a fragilidade/inexistência de fluxos, protocolos e existência/inexistência de linhas de cuidado estruturadas para a oferta do cuidado integral, principalmente no seguimento da criança exposta à sífilis e na adesão das parcerias sexuais das gestantes ao diagnóstico e tratamento.

Com a identificação e a classificação dos nós críticos expostos no quadro 2, deu-se 
início à construção de uma agenda conjunta pautada na realização do diagnóstico, com planejamento, monitoramento e avaliação regional, com vista à proposição de ações de saúde pública consistente e consoante com as necessidades de saúde das populações, com o foco no combate à sífilis, respeitando as peculiaridades de cada território. Ao mesmo tempo, as apoiadoras trouxeram para a mesa de discussão as similaridades e potencialidades de cada município em observância às diretrizes do Projeto "Sífilis Não". Desse movimento de busca por uma articulação conjunta das apoiadoras, suscitou a necessidade de envolver também as áreas técnicas dos municípios nessa articulação, o que propiciou a realização de várias oficinas regionais no período de dezembro de 2018 a dezembro de 2019, no ano de 2020. A agenda de continuidade das oficinas não se efetivou em função da pandemia da covid-19.

\section{ATORES ENVOLVIDOS}

Apoiadoras do Projeto Resposta Rápida à Sífilis nas Redes de Atenção para os municípios da Baixada Fluminense.

Representantes das áreas técnicas de Atenção Básica, Infecções Sexualmente Transmissíveis, Vigilância Epidemiológica em Saúde dos municípios que compõem a Baixada Fluminense;

Representantes das áreas técnicas de Atenção Primária em Saúde, Infecções Sexualmente Transmissíveis, Vigilância Epidemiológica em Saúde, Saúde da Mulher, da Criança e do Adolescente da Secretaria Estadual de Saúde, SES-RJ.

Supervisão do Projeto Resposta Rápida à Sífilis nas Redes de Atenção.

Secretários Municipais de Saúde dos munícipios da Baixada Fluminense, contemplados pelo Projeto.

Quadro 1 - Questionário

\section{MUNICÍPIO:}

1. Existe no seu município comitê e/ou comissão de investigação da transmissão vertical da Sífilis?

2. Como ocorre a investigação de casos?

3. Existem outras ações de investigação? Em caso positivo, descrever as ações.

4. Quais as maiores dificuldades encontradas para o desenvolvimento do trabalho de investigação da Sífilis?

5. Existe algum plano de capacitação e/ou necessidade de capacitação de equipe para o desenvolvimento deste trabalho?

6. Este município conseguiu desenvolver as ações previstas no plano de enfrentamento da sífilis? Quais as principais dificuldades?

7. Este município possui equipe qualificada para o desenvolvimento dos trabalhos?

8. Onde se encaixa no organograma o setor de investigação da sífilis neste município?

9. Existem outras instituições parceiras na investigação da sífilis que atuam junto à SMS do município (ONGs, comunidades religiosas, conselhos etc.)? Quais?

10. Qual o fluxo da investigação da sífilis do diagnóstico até o tratamento?

Profissionais responsáveis pelo preenchimento:

Fonte: Questionário elaborado pelas apoiadoras do Projeto Resposta Rápida à Sífilis com base nas informações solicitadas pela supervisão do Projeto. 
Quadro 2 - eixos/nós críticos

\begin{tabular}{|c|c|}
\hline ORDEM & SERVIÇO DE SAÚDE \\
\hline \multirow{15}{*}{ ESTRUTURAIS } & Baixa cobertura da Atenção Básica, especialmente da ESF; \\
\hline & Parte das UBS ainda com estrutura física inadequada; \\
\hline & $\begin{array}{l}\text { Número de refrigeradores e insumos para manejo de situações de urgência e } \\
\text { emergência insuficiente na rede básica; }\end{array}$ \\
\hline & Testagem rápida pouco descentralizada; \\
\hline & Distribuição e dispensação de penicilina benzatina pouco descentralizada; \\
\hline & $\begin{array}{l}\text { Baixa administração financeira dos municípios para elaboração de material } \\
\text { educativo e informativo, bem como de compra de insumos; }\end{array}$ \\
\hline & $\begin{array}{l}\text { Articulação insuficiente e/ou fragilizada entre a Atenção Básica, Especializada e } \\
\text { Hospitalar; }\end{array}$ \\
\hline & $\begin{array}{l}\text { Articulação insuficiente e/ou fragilizada entre a Atenção Básica e a Vigilância em } \\
\text { Saúde; }\end{array}$ \\
\hline & Articulação insuficiente e/ou fragilizada entre as gestões municipais e estadual; \\
\hline & $\begin{array}{l}\text { Baixa articulação com segmentos da população chave (Profissionais do sexo, } \\
\text { moradores de rua, entre outros); }\end{array}$ \\
\hline & Baixa articulação com segmentos da sociedade civil organizada; \\
\hline & Articulação intersetorial insuficiente e/ou fragilizada; \\
\hline & $\begin{array}{l}\text { Articulação insuficiente e/ou fragilizada com os Conselhos Municipais e com o } \\
\text { Conselho Estadual de Saúde; }\end{array}$ \\
\hline & $\begin{array}{l}\text { Inexistência de comitês de investigação de transmissão vertical ou de incorporação } \\
\text { da investigação dos casos de transmissão vertical pelos comitês de investigação de } \\
\text { óbito materno/fetal/infantil; }\end{array}$ \\
\hline & $\begin{array}{l}\text { Fragilidade dos processos que envolvem informação epidemiológica (notificação, } \\
\text { investigação, encerramento dos casos); }\end{array}$ \\
\hline \multirow{8}{*}{$\begin{array}{l}\text { RECURSOS } \\
\text { HUMANOS }\end{array}$} & Alta rotatividade de profissionais da gestão municipal e das equipes de saúde; \\
\hline & $\begin{array}{l}\text { Descontinuidade das chefias (exonerações) de todos os Cargos Comissionados em } \\
\text { alguns municípios por ordem do MP; }\end{array}$ \\
\hline & $\begin{array}{l}\text { Equipes técnicas das gestões municipais e equipes de saúde fragilizadas } \\
\text { tecnicamente; }\end{array}$ \\
\hline & Quantitativo insuficiente de profissionais de gestão; \\
\hline & $\begin{array}{l}\text { Equipes da Atenção Básica e Saúde da Família sobrecarregada, especialmente o } \\
\text { profissional enfermeiro; }\end{array}$ \\
\hline & $\begin{array}{l}\text { Resistência e/ou insegurança dos profissionais de enfermagem para a } \\
\text { administração da penicilina; }\end{array}$ \\
\hline & $\begin{array}{l}\text { Profissionais de saúde, em geral, pouco capacitados para o cuidado integral da } \\
\text { sífilis; }\end{array}$ \\
\hline & $\begin{array}{l}\text { Ausência de continuidade de ações e atividades de educação permanente de } \\
\text { acordo com a necessidade dos profissionais da saúde; }\end{array}$ \\
\hline \multirow{6}{*}{$\begin{array}{l}\text { GESTÃO DA } \\
\text { CLIINICA }\end{array}$} & $\begin{array}{l}\text { Ausência ou insuficiência de farmacêuticos nas redes municipais de saúde para a } \\
\text { distribuição e dispensação da penicilina (a partir da exigência do Conselho Federal } \\
\text { de Farmácia); }\end{array}$ \\
\hline & Fluxos assistenciais fragilizados ou inexistentes entre os diversos pontos da rede; \\
\hline & $\begin{array}{l}\text { Ausência ou insuficiência de protocolos municipais e linhas de cuidado da sífilis } \\
\text { (em gestantes, congênita e adquirida); }\end{array}$ \\
\hline & $\begin{array}{l}\text { Ausência ou insuficiência de fluxos e protocolos para seguimento dos casos de } \\
\text { crianças expostas e/ou diagnosticadas com sífilis congênita na rede municipal: }\end{array}$ \\
\hline & Oferta insuficiente do VDRL para diagnóstico e monitoramento dos casos; \\
\hline & $\begin{array}{l}\text { Dificuldade na abordagem às parcerias sexuais ao tratamento oportuno e } \\
\text { adequado; }\end{array}$ \\
\hline
\end{tabular}

Fonte: elaborado pelos autores. 


\section{RESULTADOS E LIÇÕES APRENDIDAS}

A experiência a partir da estratégia de articulação regional para o enfrentamento da sífilis nos municípios da Baixada Fluminense propiciou o fortalecimento do vínculo entre as apoiadoras, a partir da elaboração da proposta de trabalho integrada com os representantes municipais, dando ênfase para a articulação entre Vigilância em Saúde, Atenção Básica, Especializada e Hospitalar, sobretudo na investigação dos casos de óbitos por sífilis e seguimento das crianças expostas. Nesse processo, a elaboração, a execução e o monitoramento da proposta de trabalho regional de enfrentamento à sífilis, de maneira propositiva e com encontros sistemáticos e oportunos com os técnicos estaduais e municipais, consolidou-se como um ponto fundamental para essa articulação.

Há de se considerar ainda o fortalecimento do vínculo entre os representantes municipais e estaduais no que se refere às ações direta e indireta relacionada à sífilis nos territórios. Alguns resultados também verificados foram a melhoria da comunicação e articulação entre as áreas técnicas com impacto positivo sobre os fluxos de atendimento e processos de trabalho referente ao enfrentamento da sífilis nos municípios, assim como a elaboração de instrumentos e ferramentas de gestão que favoreçam o planejamento e o monitoramento das ações pactuadas.

\section{CONSIDERAÇÕES FINAIS}

A atuação do apoiador no Projeto "Sífilis Não" tem sido uma experiência muito rica e de muito aprendizado. Percebemos que estar junto com as áreas técnicas nos municípios, seja presencial, seja virtualmente como estamos hoje em função da pandemia, faz uma grande diferença, quando comparado ao trabalho do apoio institucional estruturado em outros moldes. Aprendemos que o trabalho em equipe, quando somado às técnicas que envolvem metodologias ativas, proporciona espaços de discussões, reflexões, criação, avaliação e monitoramento capazes de se contrapor aos fatores contrários ao bom funcionamento dos serviços de saúde.

Da experiência com a articulação regional, aprendemos a exercitar a comunicação como ferramenta primordial nos processos de trabalho, bem como a importância de cruzar fronteiras, minimizar distâncias geográficas, superar os limites pessoais que muitas vezes atravancam nossas capacidades de ação. Como apoiadoras, também compartilhamos esse aprendizado com as equipes de saúde.

Hoje, podemos afirmar que, no Estado do Rio de Janeiro, a temática "Sífilis" passou a ser fortemente pautada nas discussões e nos eventos que envolvem a saúde pública. Esperamos, com a realização deste projeto, conseguir deixar nos municípios o legado da compreensão do trabalho em equipe, da articulação e comunicação entre as áreas técnicas como contribuição para a melhor fluidez dos processos de trabalho em saúde.

\section{AGRADECIMENTOS}

Inicio com nossos agradecimentos a todos os pesquisadores e profissionais que direta ou indiretamente tornaram o Projeto "Sífilis Não" viável e exequível, tão cheio de histórias, emoções, afetos e vivências de militância no SUS. Sem eles, esta experiência não teria sido possível e nem teríamos os muitos encontros produzidos.

Aos supervisores do Projeto, Adriano e Luti, pelo apoio, pela paciência e pela compreensão de nossas angústias e insatisfação no desenvolvimento dos trabalhos.

Aos apoiadores do Projeto "Sífilis Não", pelas trocas de saberes e pelos afetos no decorrer dos trabalhos. Em especial, às minhas parceiras de apoio, Brena Cerqueira, 
Michelle Sequeira e Paula Guidone, que hoje as considero grandes amigas.

Agradeço também aos profissionais e aos gestores de saúde da Metro I, pela acoIhida, pela colaboração e pela compreensão nas muitas idas e vindas aos municípios e à Secretaria Estadual de Saúde.

Para finalizar, minha gratidão a Deus, por ter colocado todos vocês em meu caminho e me proporcionado esse novo e grandioso aprendizado. 


\section{REFERÊNCIAS}

BRASIL. Ministério da Saúde. Agenda de Ações Estratégicas para Redução da Sífilis no Brasil. Brasília: Ministério da Saúde, 2017. 34 p, il.

BRASIL. Ministério da Saúde. Boletim Epidemiológico de Sífilis. Ano VI. n. 1, p. 1-43, 2020a.

BRASIL. Ministério da Saúde. Resposta Rápida à Sífilis: uma análise situacional da estratégia de apoio institucional. Brasília: Ministério da Saúde, v.51, n.42, 2020b.

CERQUEIRA, B. G. T. Avaliação e Melhoria da Qualidade da Assistência à Sífilis Gestacional da Atenção Primária à Saúde. 2018. (Dissertação de Mestrado) Universidade Federal do Rio Grande do Norte, Natal, 2018.

GOVERNO DO ESTADO DO RIO DE JANEIRO. Secretaria de Estado de Saúde. Assessoria de Planejamento. Assessoria de Regionalização. Comissão Intergestores Regional da Região Metropolitana I. Relatório do Plano Regional Integrado. Rio de Janeiro: [s.n.], 2018.

LUCAS, M. C. V. et al. A experiência do apoio institucional do projeto de resposta rápida à sífilis nas redes de atenção à saúde. Revista Brasileira de Inovação Tecnológica em saúde, v. 9, n. 2, p. 8-24, 2019. Edição Especial - Projeto Sífilis Não. Disponível em: https:// periodicos.ufrn.br/reb/article/view/18679. Acesso em: 28 out.2020.

ROBBINS, H.; FINLEY, M. Porque as equipes não funcionam, o que não deu certo e como torna-las criativas e eficientes. 2. ed. São Paulo: Campos, 1997. 